\title{
THEORETICAL DEVELOPMENTS IN BALANCE MODEL AND MODELS WITH VIRTUAL WEIGHT RESTRICTIONS
}

\author{
Masoumeh Rajabi Tanha* and Mohammadreza Alirezaee
}

\begin{abstract}
The balance model (BM) is a unit invariant model in which takes into account the imposed strategies for DMUs through adding some balance constraints to the basic DEA models in general and the CCR model in particular. Also, balance factor is calculated along with efficiency, effectiveness and similar concepts. The mentioned balance constraints belong to the virtual weight restrictions (VWRs) category. However, to date, the consequences of incorporating weight restrictions (e.g. absolute weight restrictions, assurance regions type I (ARI), assurance regions type II (ARII)) within the classical DEA models have been explored by scholars, but are not considered for virtual weight restrictions. This paper analyses properties of the balance model and subsequently the models with VWRs by an illustrative example. The results show that models with such restrictions correctly maximize the relative efficiency (RE) of the assessed DMU despite the fact that none of the DMUs might be fully efficient (i.e. with efficiency score of 1). In addition, feasibility conditions are discussed. Finally, the proposed method will be applied to assess the branches of a specialized bank of Iran as a real application.
\end{abstract}

Mathematics Subject Classification. 90C08.

Received December 25, 2019. Accepted July 30, 2020.

\section{INTRODUCTION}

Data envelopment analysis (DEA) as a common technique assesses the relative efficiency of decision making units (DMUs) as the ratio of the weighted sum of outputs to the weighted sum of inputs without any additional prior knowledge about the prices of indices. Charnes, Cooper and Rhodes (CCR) and Banker, Charnes and Cooper (BCC) models introduced in 1978 and 1984, respectively, are the basic models in DEA literature. To date, a large number of models have been developed in different applications or in order to mitigate the existent limitations such as preventing zero weights and deleting some indices from evaluating process or improving discriminations and etc. Alirezaee and Rajabi Tanha [2] formulated a unit invariant model called a balance model (BM) and the balance factor (BF) of DMUs. This issue had been motivated not only by bank managers to assess the bank branches concerned with some predefined strategies, but also by applications of the DEA in real life organizations. The balance factor $(\mathrm{BF})$, as the ratio of CCR and the balance models, constitutes a source of additional information which reflects to what extent the assessed DMU performs along with the predefined strategies and provides deeper insights into the management. In cases where this factor equals 1 , the DMU is characterized as a balanced DMU, otherwise it is unbalanced. The lower this factor, the more the DMU

Keywords. Data envelopment analysis, balance model, virtual weight restrictions.

Iran University of Science and Technology, School of Mathematics, Resalat, Tehran, Iran.

*Corresponding author: m_rajabitanha@iust.ac.ir 
is unbalanced. Accordingly, ignoring this reality will minimize the motivation of the DMU managers who run the balanced DMUs toward the central management strategies and accept the risk of not reaching the higher possible efficiency score.

The balance factor was first expressed by Eilat et al. [10] as a ratio of the DEA-BSC score and the CCR score. Ahn et al. [1] were the first who suggested balance of DMUs as a complementary score, which avoids concentration on only some of the criteria and reduces the set of DMUs considered as benchmarks. Dyckhoff et al. [7] developed an alternative approach to consider balanced efficiency for DMUs, assuming a region within the production possibility set (PPS) in which all DMUs are $100 \%$ balanced. Dimitrov and Sutton [6] proposed a method that penalizes DMUs that use a set of asymmetric weights in their evaluation process. The aim of all these papers in introducing the balance of DMUs is to consider all criteria and not concentrate only on some. In other words, these contributions to the balance of DMUs aim at avoiding zero weights without any requirement of additional information.

The classical DEA models, which have complete flexibility to choose the optimal set of weights in order to maximize DMU's relative efficiency, may be assigned zero value to some weights of inputs or outputs and as a result these factors will be ignored in the evaluating process. So far, the most common way to avoid these problems has been to utilize several types of weight restrictions (see e.g. [3]).

Basically, weight restrictions are categorized into two main groups: those directly restricting weights (WRs) in the multiplier form of DEA models and those imposing constraints on the virtual weights (VWRs) (i.e. the product of the inputs or outputs by their related weights) [22]. The former is less complex but contains some difficulties such as depending on the units of measurement, infeasibility and sensitivity to model orientation $[3,13,14,21]$. Scholars have pointed out that virtual weight restrictions may solve the above-mentioned problems related to directly restricting weights, but none have gone through the details or carried out a deep analysis in this field $[1,11,25]$.

It should be noted that there is a similar classification of WRs for VWRs: those equivalent to absolute WRs proposed by Dyson and Thanassoulis [8] and named as absolute VWRs, and those equivalent to assurance region type I (ARI) introduced by Thompson et al. [23] called virtual assurance region type I (VARI), and assurance region type II (ARII) proposed by Thompson et al. [24] called virtual assurance region type II (VARII) [21]. Since the balance constraints placed on the balance model [2] belong to VARI, this paper focuses on this type of VWRs. The other types are left for further research. So, throughout this paper whenever we mention VWRs, it is assumed that they are from type VARI.

The mentioned balance model not only considers the experts' opinion (see also [12]) but also the indices values. Applying BM, there could be a production possibility set (PPS) in which none of the efficient DMUs are balanced, but there is an inefficient DMU which is $100 \%$ balanced. Unbalanced DMUs are panelized via placing the balance constraints to the model. Thereby, comparing CCR and BF determines the efficient but unbalanced, inefficient but balanced, inefficient and unbalanced, efficient and balanced DMUs, with the last one being the most favorable DMUs for which both CCR efficiency score and BF are equal to 1.

While incorporating restrictions, e.g. weight restrictions, may affect some properties of the basic DEA models mentioned above $[9,17,19]$, this paper analyzes these effects for the proposed balance model, which can be expanded to models with VARI restrictions. This model is unit invariant as is the model proposed by W\&B [26]. Maximin DEA model is a basic model to assess relative efficiency $[13,15,16,20]$, so in this paper we propose a generalized nonlinear form of maximin model to examine whether the incorporated weight restrictions from any type underestimate the RE or not. While, in the classical DEA model, in particular the CCR model, the relative and absolute efficiency of the assessed DMU coincide, the linear model is equivalent to maximin model. But as mentioned above, weight restrictions should be placed in the original maximin model, which explicitly maximizes the RE. This examination reveals that underestimating RE does not occur only in utilizing ARI $[13,16]$, the balance model and subsequently VARI. Also, infeasibility does not happen in the latter, provided that some trivial conditions exist. Furthermore, the mentioned models are not sensitive to the model orientation (see also [13]). 
The results show that the properties of DEA models incorporating ARI restrictions and BM and as a result VARI are the same, but there are two additional properties that should be mentioned for the latter one. While balance constraints are dependent on the assessed DMU, the PPS expansion differs from one assessed DMU to another. Of course, there are some solutions to this problem, such as considering balance constraints for all DMUs instead of just the assessed DMU, but the model becomes computationally expensive and theoretically prone to infeasibility. To overcome this computational difficulty, Wong and Beasley [26] suggested and discussed two approaches. First, applying such constraints to both the assessed DMU and the average DMU. Second, applying it only to the artificial average DMU (also, see [27]). In the first case, the problem of changing PPS while changing the assessed DMU still remains. Sometimes, it may result in all DMUs being inefficient, and note that this is different from what happens when some other kinds of WRs, such as ARII, are imposed and none are efficient, for which the frontier is constructed based on unobserved DMUs and does not include any of the observed DMUs.

The rest of the paper is organized as follows. Section 2 reviews the proposed balance model and then illustrates its properties by a numerical example. Section 3 presents a generalized model in order to check whether the relative efficiency is correctly assessed when any of the types of restrictions are added. Section 4 provides a case study on the branches of a specialized bank of Iran. Finally, Section 5 concludes the paper and provides some prospects for future research in the area of balance factor for DMUs and consequently virtual weight restrictions.

\section{THE BALANCE MODEL}

CCR was the first model proposed in data envelopment analysis (DEA) by Charnes et al. [5], and was later developed for variable returns to scale [4]. While DMUs deal with the imposed strategies, inevitably these strategies should be applied to the evaluation model. These strategies entered the proposed model as the balance constraints. The interpretation of these constraints is close to the VWRs, so these limits are used when the experts cannot predict certain bounds for the proportion of weights, but know the importance of indicators in relation to each other. In other words, these limits are used when one knows a certain level of input needed to produce a specified output. Wong and Beasley [26] were the first to introduce VWRs, as shown in (2.1).

$$
L_{r} \leq u_{r} y_{r p} / \sum_{r=1}^{s} u_{r} y_{r p} \leq U_{r}
$$

where $y_{r p}$ is $r$ th outputs of the assessed DMU (DMUp) and $u_{r}$ is $r$ th output weight. $L_{r}$ and $U_{r}$ are the lower and upper bounds of $r$ th constraint, respectively.

The formulation of balance constraints is also described in (2.2).

$$
L_{k} \leq \sum_{r \in R_{1}} u_{r} y_{r p} / \sum_{r \in R_{2}} u_{r} y_{r p} \leq U_{k} \quad \forall k, \quad R_{1}, R_{2} \subseteq\{1,2, \ldots, s\}, R_{1}, R_{2} \neq \phi
$$

where $s$ is the number of outputs and $R_{1}, R_{2}, L_{k}, U_{k}$ and $K$ (the number of balance constraints) are dependent on the imposed strategies for the assessed DMU (DMUp) and expert opinion. It is clear that W\&B constraints are a special kind of proposed balance constraint, considering $R_{1}$ as a singleton, $R_{2}=\{1,2, \ldots, \mathrm{s}\}$ and $K$ equal to the number of outputs $(K=s)$, and according to the classification of Sarrico and Dyson [21], constraints (2.2), which link virtual inputs or outputs to define their relative importance, are equivalent to VARI.

Therefore, properties of the proposed balance model (2.3) and models with VWRs, in particular VARI, are the same, and analysis of the former will lead to the latter. It is noticeable that the balance model (2.3) has been constructed with applying balance constraints (2.2) into the traditional input oriented CCR model. As mentioned in the previous section, in this paper just the VARI will be discussed and the other types, e.g. VARII 
remain for further studies.

$$
\begin{array}{ll}
\operatorname{Max} & \sum_{r=1}^{s} u_{r} y_{r p} \\
\text { s.t. } & \\
& \sum_{i=1}^{m} v_{i} x_{i p}=1 \\
& \sum_{r=1}^{s} u_{r} y_{r j}-\sum_{i=1}^{m} v_{i} x_{i j} \leq 0, \quad j=1, \ldots, n \\
& L_{k} \leq \sum_{r \in R_{1}} u_{r} y_{r p} / \sum_{r \in R_{2}} u_{r} y_{r p} \leq U_{k} \forall k, \quad R_{1}, R_{2} \subseteq\{1,2, \ldots, s\}, R_{1}, R_{2} \neq \phi \\
& u_{r} \geq \varepsilon \quad r=1, \ldots, s \\
& v_{i} \geq \varepsilon \quad i=1, \ldots, m
\end{array}
$$

where $n, m$ and $s$ are the number of DMUs, inputs and outputs, respectively. $x_{i j}, y_{r j}, v_{i}$ and $u_{r}$ are $i$ th input of $j$ th DMU, $r$ th output of $j$ th DMU, $i$ th input weight and $r$ th output weight, respectively. $\varepsilon$ is a non-Archimedean infinitesimal to avoid zero weights. In what follows, we discuss the feasibility of the balance model (2.3) and subsequently VARI.

The proposed balance model is feasible if the following conditions satisfied:

(1) Indices related to $R_{1}$ and $R_{2}$ are not zero for all members of $R_{1}$ and $R_{2}$, respectively.

(2) In the case that balance model equals to W\&B model, the sum of the lower bounds is less, and the sum of the upper bounds is greater than or equal to 1.

Proof. In this case the number of balance constraints $(K)$ equals to the number of outputs $(s)$, so in model (2.3) we have:

$$
\sum_{k=1}^{s} L_{k} \leq \sum_{r=1}^{s}\left(u_{r} y_{r p} / \sum_{r=1}^{s} u_{r} y_{r p}\right) \leq \sum_{k=1}^{s} U_{k} .
$$

Because of the following equation, the condition (2.2) would be satisfied.

$$
\sum_{r=1}^{s}\left(u_{r} y_{r p} / \sum_{r=1}^{s} u_{r} y_{r p}\right)=1
$$

\subsection{Balance model with no efficient DMUs}

A 2-dimensional numerical example as shown in Table 1 demonstrates that all DMUs may be characterized as inefficient applying balance model introduced in model (2.3) and subsequently models with virtual weight restrictions.

Assume that the imposed strategies are defined through balance constraint in (2.5). Solving balance model (2.6) with the specified balance constraint yields the results shown in Table 2.

$$
0.3 \leq u_{1} y_{1 p} /\left(u_{1} y_{1 p}+u_{2} y_{2 p}\right) \leq 0.5
$$


THEORETICAL DEVELOPMENTS IN BALANCE MODEL

TABLE 1. The data set.

\begin{tabular}{llll}
\hline \hline DMU & Input & Output1 & Output2 \\
\hline DMU $A$ & 1 & 1 & 0.3 \\
DMU $B$ & 1 & 0.3 & 0.2 \\
DMU $C$ & 1 & 0.2 & 0.4 \\
DMU $D$ & 1 & 0.4 & 0.6 \\
\hline
\end{tabular}

$$
\begin{aligned}
\operatorname{Max} & u_{1} y_{1 p}+u_{2} y_{2 p} \\
\text { s.t. } & \\
& v=1 \\
& u_{1}+0.3 u_{2}-v \leq 0 \\
& 0.3 u_{1}+0.2 u_{2}-v \leq 0 \\
& 0.2 u_{1}+0.4 u_{2}-v \leq 0 \\
& 0.4 u_{1}+0.6 u_{2}-v \leq 0 \\
& 0.3 \leq u_{1} y_{1 p} /\left(u_{1} y_{1 p}+u_{2} y_{2 p}\right) \leq 0.5 \\
& u_{1}, u_{2}, v \geq \varepsilon .
\end{aligned}
$$

With the balance constraints (2.5), the balance model (2.6) for assessing DMUs $A$ and $D$ comes in the forms below:

$$
\begin{aligned}
\operatorname{Max} & u_{1}+0.3 u_{2} \\
\text { s.t. } & \\
& u_{1}+0.3 u_{2} \leq 1 \\
& 0.3 u_{1}+0.2 u_{2} \leq 1 \\
& 0.2 u_{1}+0.4 u_{2} \leq 1 \\
& 0.4 u_{1}+0.6 u_{2} \leq 1 \\
& 0.3 \leq u_{1} /\left(u_{1}+0.3 u_{2}\right) \leq 0.5 \\
& u_{1}, u_{2}, v \geq \varepsilon \\
\operatorname{Max} & 0.4 u_{1}+0.6 u_{2} \\
\text { s.t. } & \\
& u_{1}+0.3 u_{2} \leq 1 \\
& 0.3 u_{1}+0.2 u_{2} \leq 1 \\
& 0.2 u_{1}+0.4 u_{2} \leq 1 \\
& 0.4 u_{1}+0.6 u_{2} \leq 1 \\
& 0.3 \leq 0.4 u_{1} /\left(0.4 u_{1}+0.6 u_{2}\right) \leq 0.5 \\
& u_{1}, u_{2}, v \geq \varepsilon .
\end{aligned}
$$

The feasible regions are different for each assessed DMU, as portrayed in weight spaces with shaded areas for DMUs $A$ and $D$ in Figures 1 and 2. The feasible regions are the intersection between the space defined by the constraints relevant to each DMU and the cone defined by the balance constraints. While the balance 
TABLE 2. Results.

\begin{tabular}{lllllll}
\hline \hline DMU & BM & $v$ & $u_{1}$ & $u_{2}$ & CCR & BF \\
\hline DMU A & 0.83 & 1 & 0.417 & 1.389 & 1 & 0.83 \\
DMU B & 0.44 & 1 & 0.625 & 1.25 & 0.44 & 1 \\
DMU C & 0.49 & 1 & 0.741 & 0.864 & 0.67 & 0.74 \\
DMU D & 0.91 & 1 & 0.682 & 1.061 & 1 & 0.91 \\
\hline
\end{tabular}

Notes. "BM" refers to the solution of model (2.6), and "CCR" refers to the solution of the traditional DEA input oriented model, that corresponds to model (2.6) excluding the balance constraints.

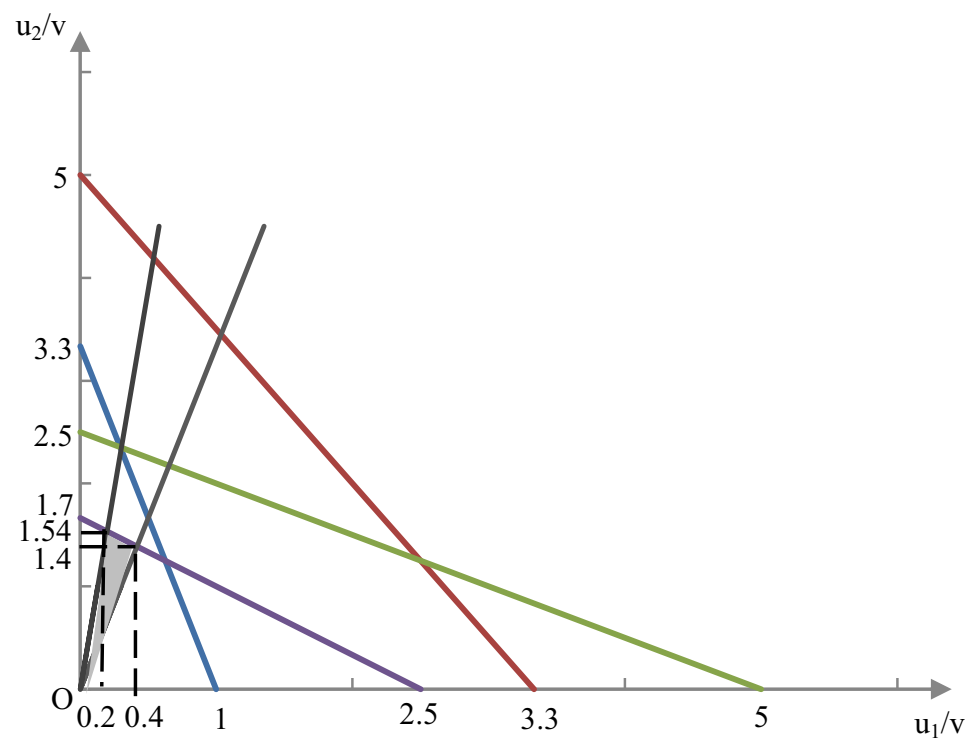

Figure 1. Feasible region of model (2.7) for DMU $A$.

constraints depend on the assessed DMUs, the feasible regions differ from one to another. Results of balance model (2.6) in Table 2 show that none of the DMUs is efficient. This is not because of the underestimation that happens when incorporating ARII or absolute WRs. In the evaluation process of assessed DMU, certainly there is a DMU with $100 \%$ efficiency for the optimal weights of the assessed DMU (which is the reason for correctly estimating relative efficiency of classical DEA models and models with ARI restrictions), in other words, because the feasible region meets at least one of the constraints relevant to DMUs, so undoubtedly one of them is assigned efficiency equal to 1 . The point here is that these weights may become infeasible, assessing the efficient DMU, and one of the other DMUs would be assigned the efficiency equal to 1.

Figures 3 and 4 provide a clear picture of the PPS in the output space in which outputs have been normalized by the input, for DMUs $A$ and $D$ respectively, to illustrate the reason for efficiency of less than one for all DMUs. Given that DMUs $B$ and $C$ are inefficient, solving the CCR model, they do not affect construction of the PPS frontier and we will just discuss DMUs $A$ and $D$. Assessing DMU $A$, DMU $D$ is on the efficient frontier, and assessing DMU $D$, DMU $A$ is put on the frontier. This happens just because of differing PPS from one assessed DMU to another and not from underestimating RE or other probable reasons. In other words, using this kind of restriction, only the DMU under assessment is required to respect the constraints on the virtual weights, and other DMUs that are not facing the weight restrictions may take advantage of this and be classified as efficient. 


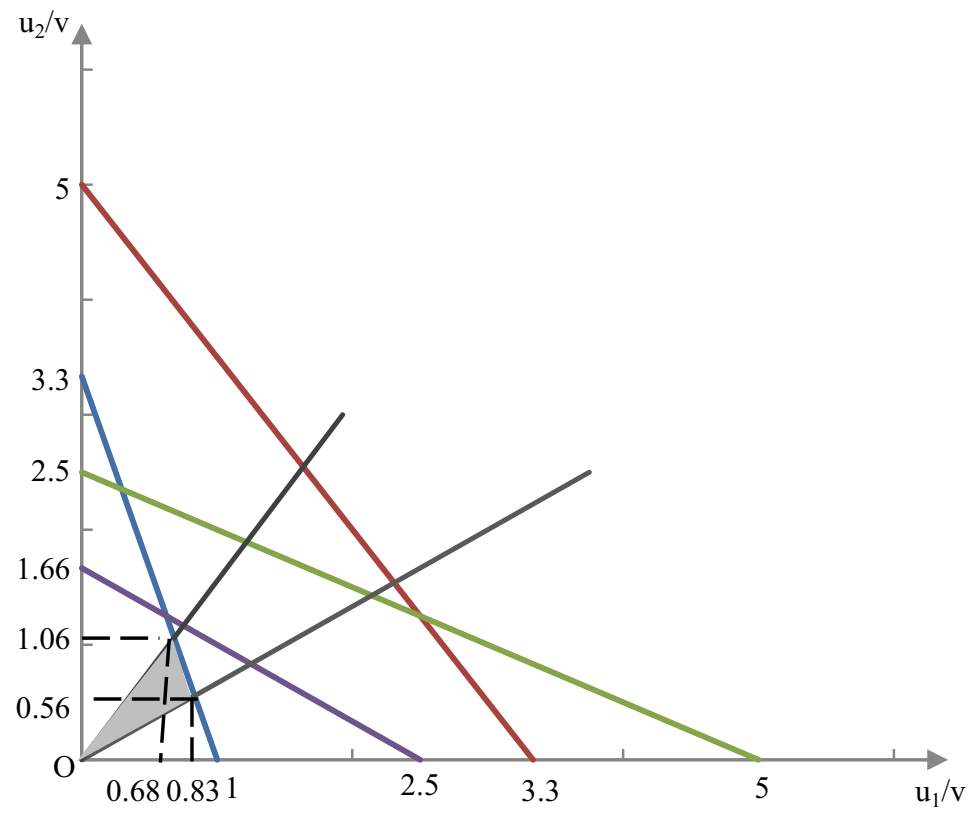

Figure 2. Feasible region of model (2.8) for DMU D.

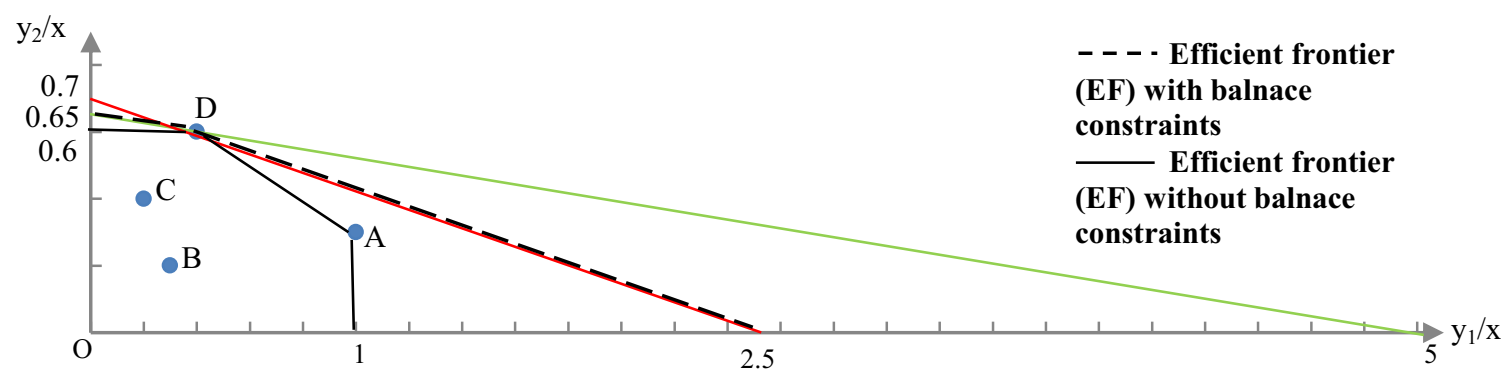

FIGURE 3. The efficient frontier with and without balance constraint (2.5) for DMU $A$.

Now considering the envelopment form of model (2.6), which is to some extent in the form of DEA models with trade-offs proposed by Podinovski [17-19], differing PPS from one assessed DMU to another can be shown as well.

$$
\begin{array}{ll}
\min & \theta \\
\text { s.t. } & \\
& \sum_{j=1}^{4} \lambda_{j} x_{1 j} \leq \theta x_{1 p} \\
& \sum_{j=1}^{4} \lambda_{j} y_{1 j}-0.7 y_{1 p} \pi_{1}+y_{1 p} \pi_{2} \geq y_{1 p}
\end{array}
$$




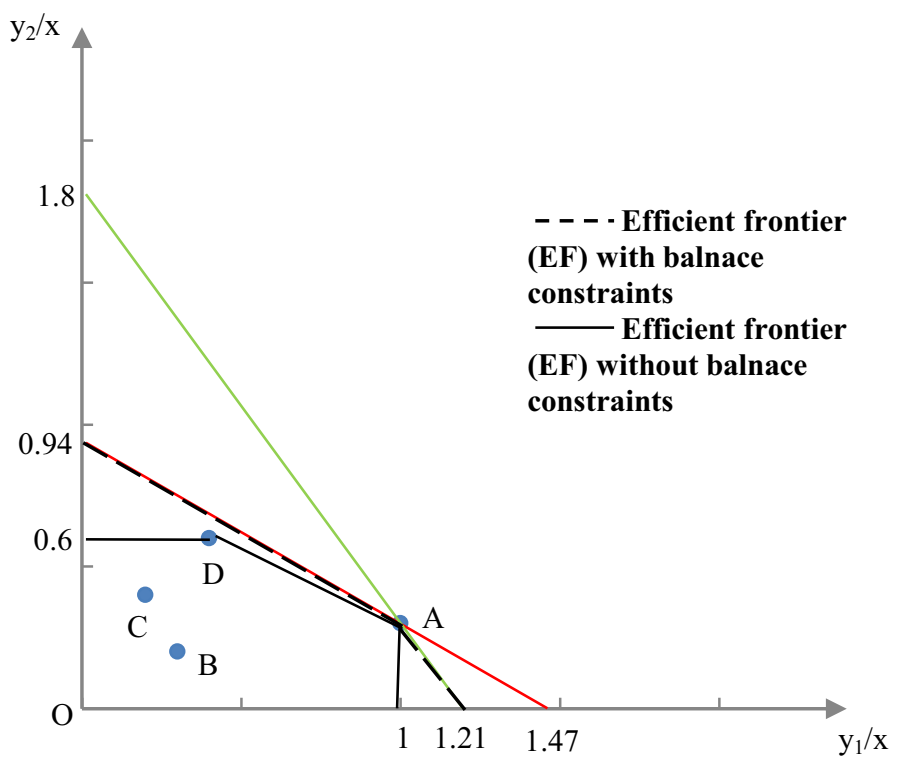

FIgURE 4. The efficient frontier with and without balance constraint (2.5) for DMU $D$.

$$
\begin{aligned}
& \sum_{j=1}^{4} \lambda_{j} y_{2 j}+0.3 y_{2 p} \pi_{1}-y_{2 p} \pi_{2} \geq y_{2 p} \\
& \lambda_{j} \geq 0 \quad \forall j \\
& \pi_{1}, \pi_{2} \geq 0
\end{aligned}
$$

where $\theta$ is a positive variable. As follows from model (2.9), the trade-offs induced by the balance constraints (2.5) which are represented in (2.10) are dependent on the assessed DMU as they are multiplied by $Y_{p}$ (i.e. output values of the assessed DMU) which results in different PPS for different under-evaluated DMUs. It is notable that this dependency occurs when non-homogenous weight restrictions are applied as well.

$$
T_{1}:\left(0,-0.7 y_{1 p}, 0.3 y_{2 p}\right), \quad T_{1}:\left(0, y_{1 p},-y_{2 p}\right) .
$$

\section{The proposed Generalized model to GuARAntee Assessing the Correct RE}

The results of balance model presented in the previous section with no efficient DMUs prompt us to investigate if the relative efficiency scores are correctly estimated or not.

The fractional and linear CCR model assesses the absolute efficiency of DMUs, which is equal to their relative efficiency. However, as adding weight restrictions may cause underestimation of the relative efficiency, it is proposed to utilize the maximin DEA model, in which its objective function is the relative efficiency and to place these restrictions into this model (see also [15]). In this section, a generalized nonlinear model (GM) shown in (3.1) is proposed to examine whether the models with incorporated restrictions from any type correctly estimate the RE. This examination of the proposed balance model and models in the presence of VARI reveals that they successfully estimate the relative efficiency of DMUs. Table 3 shows the result of GM (model (3.1)) with balance constraint (2.5) and balance model (2.6) which balance constraint (2.5) has been added to linear form of maximin model. As shown, the results are the same, so the underestimation does not have occurred. Since the VARI is a special kind of introduced balance constraints, so this type of restriction does not underestimate 
TABLE 3. Results.

\begin{tabular}{lll}
\hline \hline DMU & BM & GM with $\mathrm{BC}^{*}$ \\
\hline DMU $A$ & 0.83 & 0.83 \\
DMU $B$ & 0.44 & 0.44 \\
DMU $C$ & 0.49 & 0.49 \\
DMU $D$ & 0.91 & 0.91 \\
\hline
\end{tabular}

Notes. ${ }^{*}$ This column is the results of model (3.1) with balance constraint (2.5).

$\mathrm{RE}$ as well.

$$
\begin{array}{ll}
\operatorname{Max} & t \sum_{r=1}^{s} u_{r} y_{r p} \\
\text { s.t. } & \\
& t c \sum_{i=1}^{m} v_{i} x_{i p}=1 \\
& \sum_{r=1}^{s} u_{r} y_{r j}-c \sum_{i=1}^{m} v_{i} x_{i j} \leq 0, \quad j=1, \ldots, n \\
& u_{r}, v_{i} \geq \varepsilon \quad r=1, \ldots, s, i=1, \ldots, m \\
& c, t \geq \varepsilon
\end{array}
$$

where $t$ and $c$ are positive variables.

\section{Proof of equivalence between maximin model and model (3.1)}

Starting from Maximin model (1) (see [13]) and defining the positive variable $c=\operatorname{Max}_{j} \sum_{r=1}^{s} u_{r} y_{r j} / \sum_{i=1}^{m} v_{i} x_{i j}$, we obtain model (2) as follows:

$$
\mathrm{RE}_{p}=\operatorname{Max}_{u . v} \frac{\frac{\sum_{r=1}^{s} u_{r} y_{r p}}{\sum_{i=1}^{m} v_{i} x_{i p}}}{\operatorname{Max}_{j} \frac{\sum_{r=1}^{s} u_{r} y_{r j}}{\sum_{i=1}^{m} v_{i} x_{i j}}} \quad(1) \quad \rightarrow \quad \text { s.t. } \begin{aligned}
& \operatorname{Max} \frac{\sum_{r=1}^{s} u_{r} y_{r p}}{c \sum_{i=1}^{m} v_{i} x_{i p}} \\
& \frac{\sum_{r=1}^{s} u_{r} y_{r j}}{\sum_{i=1}^{m} v_{i} x_{i j}} \leq c, \quad j=1, \ldots, n .
\end{aligned}
$$

Then, we can obtain model (3) as shown below:

$$
\begin{array}{ll}
\operatorname{Max} & \frac{\sum_{r=1}^{s} u_{r} y_{r p}}{c \sum_{i=1}^{m} v_{i} x_{i p}} \\
\text { s.t. } & \\
& \sum_{r=1}^{s} u_{r} y_{r j}-c \sum_{i=1}^{m} v_{i} x_{i j} \leq 0, \quad j=1, \ldots, n .
\end{array}
$$

Now defining $c \sum_{i=1}^{m} v_{i} x_{i p}=1 / t$, we obtain the proposed generalized model (3.1).

Model (3.1) can be simplified in special cases. For instance, applying ARII restrictions $t$ can be assumed 1 (i.e. it can be omitted), and in cases where ARI restrictions are imposed on the model, both $t$ and $c$ can be ignored because GM is identical to the basic CCR model. But regarding absolute weight restrictions, both 
should be considered in order to estimate the correct relative efficiency. The output-oriented form of model (3.1) is represented in (3.2) as well.

$$
\begin{array}{ll}
\text { Min } & t \sum_{i=1}^{m} v_{i} x_{i p} \\
\text { s.t. } & \\
& t c \sum_{r=1}^{s} u_{r} y_{r p}=1 \\
& c \sum_{r=1}^{s} u_{r} y_{r j}-\sum_{i=1}^{m} v_{i} x_{i j} \leq 0, \quad j=1, \ldots, n \\
& u_{r}, v_{i} \geq \varepsilon \quad r=1, \ldots, s, i=1, \ldots, m \\
& c, t \geq \varepsilon .
\end{array}
$$

\section{An APPLiCATiOn TO THE BANK BRANCHES}

In this section we will apply the proposed balance model (2.3) to assess 194 branches of a specialized bank in Iran, which are concerned with some imposed strategies as already mentioned. Table 4 describes the inputs and outputs used in the proposed model for the bank branches, and Table 5 summarizes the descriptive statistics of the input and output data.

Personnel expenses and location are two inputs of branches in this study. Whereas, resources, consumptions, and services are considered as their three outputs.

The input of personnel expenses includes all the quantities such as the hours of each person worked in a month, the extra hours of each person worked, etc. and also qualities entities related to the staff of a branch such as the experiences of each person, the knowledge of each person, and so on for all people working in a branch. The mentioned factors have affected the amount of payment to each person and the summation of the payments to the personnel is considered as the input of personnel expenses. The input of location includes all the quantities and qualities entities related to the physical location of a branch such as the general factors related to the situation of the location from a commercial, industrial, or residential point of view and also, the specific factors related to the presence of the competitive banks' branches in the location. The planning and programming department of the bank has undertaken a project for this index and they have considered all the related factors in the developed location index. Therefore, we used the data of the location index in the evaluation.

The output of resources includes all kinds of methods of gathering money by a branch. A project has been undertaken for calculating this index by the planning and programming department of the bank as well. They used a weighted sum of all kinds of accounts considering their values and number of transactions for calculation of the deposit index and we used the data of the resources index in our evaluation. The output of consumptions includes all the money going out from a branch as all kinds of loans and mortgages. Similar to resources index some calculations have been done. Finally, the output of services is an index which includes all kinds of services presented by a branch to its customers.

The imposed strategies are defined in (4.1) as balanced constraints.

$$
0.5 \leq u_{1} y_{1 p} /\left(u_{2} y_{2 p}+u_{3} y_{3 p}\right) \leq 4.5, \quad 0.67 \leq u_{2} y_{2 p} / u_{3} y_{3 p} \leq 6 .
$$

The bounds in the balanced constraints of (4.1) are built based on the following two strategies of the bank board of direction:

Strategy 1: the value of the resources in the evaluation must be as much as half of the summation of the values of the consumptions and the services at least, and also, it must be as much as 4.5 times of the summation of the values of the consumptions and the services at most. 
TABLE 4. Inputs and Outputs of the branches.

\begin{tabular}{ll}
\hline \hline Inputs & Outputs \\
\hline Personnel expenses & Resources \\
Location of branch & $\begin{array}{l}\text { Consumptions } \\
\text { Services }\end{array}$ \\
\hline
\end{tabular}

TABLE 5. Descriptive statistics for the data.

\begin{tabular}{lllll}
\hline \hline & Min & Max & Average & STD \\
\hline $\begin{array}{l}\text { Inputs } \\
\begin{array}{l}\text { Personnel expenses } \\
\text { Location of branch }\end{array}\end{array}$ & 576.1000 & 1212.0000 & 965.1438 & 143.0668 \\
$\begin{array}{l}\text { Outputs } \\
\text { Resources }\end{array}$ & & & & \\
consumptions & 75.560 & 18288.000 & 1950.373 & 2231.807 \\
services & 6.738 & 8615.000 & 1313.151 & 1320.460 \\
\hline
\end{tabular}

Notes. The personnel expenses index is in terms of 10000 Rials, and the other indices have no units which are normalized, so that the summation of each index for all branches of the bank is 1000000 .

Strategy 2: the value of the consumptions in the evaluation must be as much as one third of the value of the services at least, and also, it must be as much as 6 times of the value of the services at most.

Model (4.2) represents the balance model with balance constraints (4.1) as follows.

$$
\begin{array}{ll}
\operatorname{Max} & \sum_{r=1}^{3} u_{r} y_{r p} \\
\text { s.t. } & \\
& \sum_{i=1}^{2} v_{i} x_{i p}=1 \\
& \sum_{r=1}^{3} u_{r} y_{r j}-\sum_{i=1}^{2} v_{i} x_{i j} \leq 0, \quad j=1, \ldots, 194 \\
& 0.5 \leq u_{1} y_{1 p} /\left(u_{2} y_{2 p}+u_{3} y_{3 p}\right) \leq 4.5 \\
& 0.67 \leq u_{2} y_{2 p} / u_{3} y_{3 p} \leq 6 \\
& u_{r} \geq \varepsilon \quad r=1, \ldots, s \\
& v_{i} \geq \varepsilon \quad i=1, \ldots, m .
\end{array}
$$

Results of balance model (4.2), the efficiency scores of CCR (model (4.2) excluding balance constraints (4.1)) and balance factor (the ratio of BM to CCR scores) of the branches, are summarized for some of the branches as a sample (see Tab. 6). As the results depict, two branches are CCR efficient, but none are fully efficient while applying BM (4.2). Consequently, comparing CCR and BF demonstrates that branches 113 and 179 are efficient but unbalanced and the second is comparatively more unbalanced. Branches 42 and 92 are balanced according to the table but they are inefficient. Other branches are inefficient and unbalanced, and with the scores obtained from the proposed method, it is determined that to what extent the branches are inefficient and unbalanced in order to help making decisions for branches. 
TABLE 6. The results.

\begin{tabular}{llll}
\hline \hline Branches & CCR model & Balance model $(\mathrm{BM})$ & Balance factor $(\mathrm{BF})$ \\
\hline 113 & 1.00 & 0.93 & 0.93 \\
179 & 1.00 & 0.31 & 0.31 \\
23 & 0.87 & 0.85 & 0.98 \\
2 & 0.86 & 0.71 & 0.83 \\
6 & 0.83 & 0.80 & 0.96 \\
88 & 0.82 & 0.76 & 0.93 \\
11 & 0.75 & 0.58 & 0.78 \\
14 & 0.73 & 0.70 & 0.96 \\
$\vdots$ & & & \\
42 & 0.24 & 0.24 & 1.00 \\
$\vdots$ & & & \\
92 & 0.23 & 0.23 & \\
$\vdots$ & & & \\
\hline
\end{tabular}

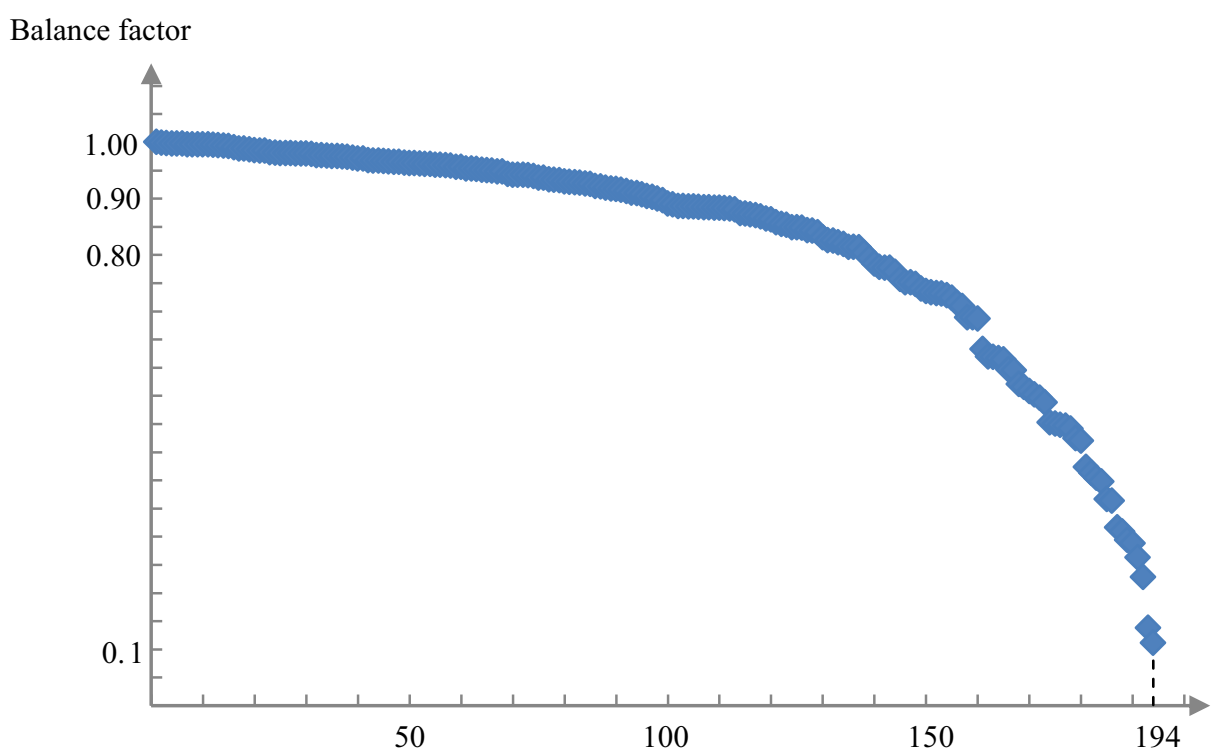

FiguRE 5. Balance factor for 194 branches.

Figure 5 portrays the distribution of branches with respect to the balance factor, and as shown the balance factors of more than $50 \%$ of branches are greater than $90 \%$ and this shows that most of them try to be aligned with the imposed strategies, and the balance factors of about $20 \%$ of branches are less than $50 \%$.

\section{Findings AND FUture PROSPECTS}

The balance model (BM) which takes into account the imposed strategies on DMUs and the balance factor which describes to what extent the assessed DMU performs along with these strategies are reviewed. In this paper, the properties of BM were discussed and feasibility and underestimating the relative efficiency which is 
the main property of DEA models were investigated through an illustrative example. The results showed that this model does not have the problem of underestimation, despite none of DMUs are fully efficient. Thereafter a generalized nonlinear form of maximin model proposed to examine this property for every model with different types of restrictions. Then the balance model was applied to a real-world application. Some issues have been left for further studies, for instance the cases in which balance constraints are from VARII type. Moreover, we believe that restricting virtual weights in DEA is applicable, in particular in variable returns to scale for which other forms of weight restrictions are not suitable. This is due to the fact that they explicitly assume that all DMUs should accept the weight restrictions, whereas in variable returns to scale, the imposed WRs may not be valid for different sizes and some other weight restrictions dependent on scale of DMUs should be utilized. VWRs are proper choices in these circumstances.

\section{REFERENCES}

[1] H. Ahn, L. Neumann and N. Vazquez Novoa, Measuring the relative balance of DMUs. Eur. J. Oper. Res. 221 (2012) 417-423 [corrigendum: Eur. J. Oper. Res. 222 (2012) 686.].

[2] M.R. Alirezaee and M. Rajabi Tanha, Extending the Malmquist index to consider the balance factor of decision making units in a productivity analysis. IMA J. Manage. Math. 27 (2016) 439-452.

[3] R. Allen, A. Athanassopoulos, R.G. Dyson and E. Thanassoulis, Weights restrictions and value judgments in data envelopment analysis: evolution development and future directions. Ann. Oper. Res. 73 (1997) 13-34.

[4] R.D. Banker, A. Charnes and W.W. Cooper, Some models for estimating technical and scale inefficiency in data envelopment analysis. Manage. Sci. 31 (1984) 1078-1092.

[5] A. Charnes, W.W. Cooper and E. Rhodes, Measuring the efficiency of decision making units. Eur. J. Oper. Res. 2 (1978) 429-444.

[6] S. Dimitrov and W. Sutton, Generalized symmetric weight assignment technique: incorporating managerial preferences in data envelopment analysis using a penalty function. Omega 41 (2013) 48-54.

[7] H. Dyckhoff, M. Clermont, A. Dirksen and E. Mbock, Measuring balanced effectiveness and efficiency of German business schools research performance. In: Performance Management im Hochschulbereich, edited by Alexander Dilger, Harald Dyckhoff and Günter Fandel. Springer Gabler (2013) 39-60.

[8] R.G. Dyson and E. Thanassoulis, Reducing weight flexibility in data envelopment analysis. J. Oper. Res. Soc. 39 (1988) $563-576$.

[9] R.G. Dyson, R. Allen, A.S. Camanho, V.V. Podinovski, C.S. Sarrico and E.A. Shale, Pitfalls and protocols in DEA. Eur. J. Oper. Res. 132 (2001) 245-259.

[10] H. Eilat, B. Golany and A. Shtub, Constructing and evaluating balanced portfolios of R\&D projects with interactions: a DEA based methodology. Eur. J. Oper. Res. 172 (2006) 1018-1039

[11] M.P. Estellita Lins, A.C. Moreira da Silva, C.A.K. Lovell, Avoiding infeasibility in DEA models with weight restrictions. Eur. J. Oper. Res. 181 (2007) 956-966.

[12] M. Halme, T. Joro, P. Korhonen, S. Salo and J. Wallenius, Avalue efficiency approach to incorporating preference information in data envelopment analysis. Manage. Sci. 45 (1999) 103-115.

[13] M. Khalili, A.S. Camanho, M.C.A.S. Portela and M.R. Alirezaee, The measurement of relative efficiency using data envelopment analysis with assurance regions that link inputs and outputs. Eur. J. Oper. Res. 203 (2010) 761-770.

[14] F. Pedraja-Chaparro, J. Salinas-Jimenez and P. Smith, On the role of weight restrictions in data envelopment analysis. J. Prod. Anal. 8 (1997) 215-230.

[15] V.V. Podinovski, Side effects of absolute weight bounds in DEA models. Eur. J. Oper. Res. 115 (1999) $583-595$.

[16] V.V. Podinovski, DEA models for explicit maximization of relative efficiency. Eur. J. Oper. Res. 52 (2001) 572-586.

[17] V.V. Podinovski, Production trade-offs and weight restrictions in data envelopment analysis. J. Oper. Res. Soc. 55 (2004) $1311-1322$.

[18] V.V. Podinovski, Computation of efficient targets in DEA models with production trade-offs and weight restrictions. Eur. J. Oper. Res. 181 (2007) 586-591.

[19] V.V. Podinovski, Optimal weights in DEA models with weight restrictions. Eur. J. Oper. Res. 254 (2016) $916-924$.

[20] V.V. Podinovski and A. Athanassopoulos, Assessing the relative efficiency of decision making units using DEA models with weight restrictions. J. Oper. Res. Soc. 49 (1998) 500-508.

[21] C.S. Sarrico and R.G. Dyson, Restricting virtual weights in data envelopment analysis. Eur. J. Oper. Res. 159 (2004) 17-34.

$[22]$ E. Thanassoulis, M.C.A.S. Portela and O. Despic, Data envelopment analysis: the mathematical programming approach to efficiency analysis. In: The Measurement of Productive Efficiency and Productivity Growth, edited by H.O. Fried, C.A.K. Lovell and S.S. Schmidt. Oxford University Press, New York (2008) 251-420.

[23] R.G. Thompson, F.D. Singleton, R.M. Thrall and B.A. Smith, Comparative site evaluations for locating a high-energy physics lab in Texas. Interfaces 16 (1986) 35-49.

[24] R.G. Thompson, L.N. Langemeier, C. Lee, E. Lee and R.M. Thrall, The role of multiplier bounds in efficiency analysis with application to Kansas farming. J. Econom. 46 (1990) 93-108. 
[25] D.L. Tracy and B. Chen, A generalized model for weight restrictions in data envelopment analysis. J. Oper. Res. Soc. 56 (2005) 390-396.

[26] Y.-H.B. Wong and J.E. Beasley, Restricting weight flexibility in data envelopment analysis. J. Oper. Res. Soc. 41 (1990) 829-835.

[27] A. Zanella, A.S. Camanho and T.G. Dias, Undesirable outputs and weighting schemes in composite indicators based on data envelopment analysis. Eur. J. Oper. Res. 245 (2015) 517-530. 